\title{
Efficacy of pirfenidone in patients with idiopathic pulmonary fibrosis with more preserved lung function
}

\author{
Carlo Albera1, Ulrich Costabel ${ }^{2}$, Elizabeth A. Fagan³, Marilyn K. Glassberg ${ }^{4}$, \\ Eduard Gorina ${ }^{3}$, Lisa Lancaster ${ }^{5}$, David J. Lederer ${ }^{6}$, Steven D. Nathan ${ }^{7}$, \\ Dominique Spirig ${ }^{8}$ and Jeff J. Swigris ${ }^{9}$
}

Affiliations: ${ }^{1}$ Dept of Clinical and Biological Sciences, University of Turin, Turin, Italy. ${ }^{2}$ Ruhrlandklinik, University Hospital, University of Duisburg - Essen, Essen, Germany. ${ }^{3}$ Clinical Science, InterMune Inc., Brisbane, CA, USA. ${ }^{4}$ University of Miami Miller School of Medicine, Miami, FL, USA. ${ }^{5}$ Division of Allergy, Pulmonary and Critical Care Medicine, Vanderbilt University Medical Center, Nashville, TN, USA. ${ }^{6}$ Depts of Medicine and Epidemiology, Columbia University Medical Center, New York, NY, USA. ${ }^{7}$ Dept of Medicine, Inova Fairfax Hospital, Falls Church, VA, USA. ${ }^{8}$ Global Medical Affairs, F. Hoffmann-La Roche Ltd, Basel, Switzerland. ${ }^{9}$ Interstitial Lung Disease Program, Division of Pulmonary, Critical Care, and Sleep Medicine, Dept of Medicine, National Jewish Health, Denver, CO, USA.

Correspondence: Carlo Albera, Dept of Clinical and Biological Sciences, Interstitial and Rare Lung Diseases Unit, University of Turin, School of Medicine, San Luigi Gonzaga Hospital, Regione Gonzole 10, 10043 Orbassano, Turin, Italy. E-mail: carlo.alberadyahoo.it

ABSTRACT This post hoc analysis examined the differences in idiopathic pulmonary fibrosis disease progression and the effects of pirfenidone in patients stratified by more preserved versus less preserved baseline lung function status using forced vital capacity (FVC) or GAP (gender, age and physiology) index stage.

Efficacy outcomes, i.e. FVC, 6-min walking distance (6MWD) and dyspnoea (University of California San Diego Shortness of Breath Questionnaire (UCSD SOBQ)), were analysed at 12 months in patients randomised to pirfenidone $2403 \mathrm{mg} \cdot \mathrm{day}^{-1}$ or placebo in the pooled phase 3 CAPACITY/ASCEND population ( $n=1247$ ), with subgroups stratified by baseline $\mathrm{FVC} \geqslant 80 \%$ versus $<80 \%$ or GAP stage I versus II-III. Treatment-by-subgroup interaction was tested based on a rank ANCOVA model; factors in the model included study, region, treatment, subgroup and treatment-by-subgroup interaction term.

Patients with both more preserved (FVC $\geqslant 80 \%$ or GAP stage I) and less preserved (FVC $<80 \%$ or GAP stage II-III) lung function at baseline demonstrated clinically significant disease progression at 12 months in terms of categorical decline in FVC, 6MWD and UCSD SOBQ. The magnitude of pirfenidone treatment effect was comparable between subgroups, regardless of whether lung function was classified using FVC or GAP index stage.

These findings support the initiation of treatment with pirfenidone, irrespective of stage of baseline lung function in this patient population.

@ERSpublications

Pirfenidone is efficacious in patients with idiopathic pulmonary fibrosis (IPF) with more preserved lung function http://ow.ly/Ajlt300SR89

This article has supplementary material available from erj.ersjournals.com

Received: Feb 122015 | Accepted after revision: May 25 2016 | First published online: July 282016

Support statement: This study was funded by F. Hoffmann-La Roche Ltd. Funding information for this article has been deposited with the Open Funder Registry.

Conflict of interest: Disclosures can be found alongside this article at erj.ersjournals.com

Copyright OERS 2016 


\section{Introduction}

Idiopathic pulmonary fibrosis (IPF) is a chronic, progressive, irreversible and fatal lung disease with median survival of $\sim 2-5$ years from diagnosis $[1,2]$. The course of IPF is highly unpredictable with significant variation across individuals; some patients have gradual worsening of lung function over the course of years, some patients remain stable over many months to years, other patients decline rapidly from disease onset, and still others experience periods of clinical stability interrupted by episodes of acute exacerbations and respiratory worsening [3-5]. As a result, it is difficult to characterise disease progression in IPF. Similarly, there is no universal definition for IPF disease severity or staging; however, forced vital capacity (FVC) and the GAP (gender, age and physiology) index have been used for this purpose.

FVC is considered a reliable measure of disease progression in patients with IPF $[2,6]$. Despite the ongoing debate on the merits of FVC as a surrogate marker, including use of relative versus absolute decline in FVC $[7,8]$, evidence-based guidelines for the management of IPF suggest that measuring an absolute decline in $\geqslant 10 \% \mathrm{FVC}$ is an acceptable method of estimating the risk of mortality [2]. The GAP index uses four baseline variables (gender, age and physiology: FVC and diffusing capacity of the lung for carbon monoxide (DLCO)) to predict mortality risk in IPF $[9,10]$. The GAP index is not useful for predicting future decline in pulmonary function [11]; however, GAP stages I, II and III have predicted 1 -year mortalities of $6 \%, 16 \%$ and $39 \%$, respectively [10]. This simple, multidimensional index may provide a proper basis for discussing prognosis and management options, and the ability to identify at-risk populations [10]. Although the GAP index is influenced by gender and age, and was developed as an indicator of mortality risk, in the context of a 52-week study where gender does not change and age increases marginally, the GAP index is useful as a measure of lung function.

Both pirfenidone and nintedanib have been approved in multiple countries (including Europe and the USA) for the treatment of IPF. Pirfenidone is an oral antifibrotic agent with anti-inflammatory properties shown to decrease disease progression in IPF, as assessed by lung function, exercise tolerance and progression-free survival [12-14]. A pooled analysis of data from the phase 3 CAPACITY and ASCEND trials showed that pirfenidone significantly reduces all-cause mortality and treatment-emergent IPF-related mortality at 1 year [12]. Pirfenidone is generally well tolerated in patients with IPF.

In a disease such as IPF, in which the available approved treatment options delay disease progression but are not curative, intervention early in the disease course may be the appropriate strategy to preserve functional status and extend life, rather than take the "watch-and-wait" approach; however, published data to support this hypothesis are lacking. The present analysis reports the efficacy and safety outcomes at 12 months in patients treated with pirfenidone $2403 \mathrm{mg} \cdot \mathrm{day}^{-1}$ or placebo in the pooled CAPACITY and ASCEND study populations $(n=1247)$, as stratified by baseline FVC or GAP index stage. The objectives of this analysis were to better understand the differences in disease progression and to determine whether treatment with pirfenidone is beneficial regardless of baseline disease stage by examining the effects of pirfenidone in patients stratified by more preserved versus less preserved baseline lung function using FVC or GAP index stage.

\section{Patients and methods}

Data sources

In this post hoc analysis, data were pooled from patients $(n=1247)$ who were randomised to pirfenidone $2403 \mathrm{mg} \cdot \mathrm{day}^{-1}$ or placebo in the three phase 3 controlled trials, CAPACITY (PIPF-004 and PIPF-006 studies) and ASCEND (PIPF-016 study) $[12,13]$. Analyses were conducted by stratifying patients by baseline lung function using FVC (FVC $\geqslant 80 \%$ versus $<80 \%)$ or GAP index stage. Only 86 patients were classified as having GAP stage III disease (54 received pirfenidone and 32 received placebo) and could not be meaningfully analysed as a separate group; therefore, patients with GAP stage II and III disease were pooled for the purpose of this analysis (i.e. GAP stage I versus II-III). Patients with baseline FVC $\geqslant 80 \%$ predicted or GAP stage I were considered to have "more preserved" lung function, while those with baseline FVC $<80 \%$ predicted or GAP stage II-III had "less preserved" lung function.

\section{Efficacy and safety outcomes}

Efficacy outcomes were assessed at 12 months and included categorical and mean absolute decline in FVC \% predicted, 6-min walking distance (6MWD) and dyspnoea using the University of California San Diego Shortness of Breath Questionnaire (UCSD SOBQ) scores. The UCSD SOBQ is a 24-item questionnaire that assesses patient-reported shortness of breath on a six-point scale $(0=$ "not at all" to $5=$ "maximal or unable to do because of breathlessness") while performing a variety of activities of daily living [15].

Safety outcomes were recorded as treatment-emergent from the first dose up to 28 days after the last dose of study drug. The safety outcomes included treatment-emergent adverse events, treatment-emergent adverse events leading to discontinuation, treatment-emergent serious adverse events and treatment-emergent deaths. 


\section{Statistical analyses}

Efficacy outcomes in both studies were assessed every 3 months for 1 year. Months 3, 6, 9, 12 correspond to weeks 12, 24, 36 and 48 for PIPF-004 and PIPF-006, and to weeks 13, 26, 39 and 52 for PIPF-016. The baseline assessment was the mean of the two FVC measurements obtained at the screening and day 1 visits. For PIPF-016, the 12 months assessment was the mean of the two FVC measurements obtained at the two week 52 visits. Missing FVC, 6MWD and UCSD SOBQ values were imputed by using the sum of squared differences method [12].

For categorical analyses, the proportions of patients experiencing $\geqslant 10 \%$ decline in FVC or death, $\geqslant 50 \mathrm{~m}$ decline in $6 \mathrm{MWD}$ or death and worsening of $\geqslant 20$ UCSD SOBQ points or death were summarised at 12 months, and presented according to treatment group and baseline FVC/GAP index stage subgroups. An absolute decline in $\mathrm{FVC} \geqslant 10 \%$ was chosen based on previous data demonstrating a minimal clinically important difference (MCID) of $2-6 \%$ and that a decline $\geqslant 10 \%$ was associated with an 8 -fold increase in the risk of 1 -year mortality in patients with IPF $[6,16]$. Similarly, a categorical decline of $\geqslant 50$ for $6 \mathrm{MWD}$ was chosen based on previously reported MCIDs between 24 and $45 \mathrm{~m}$ and a 3 -fold increased risk of mortality for patients who demonstrated a $>50 \mathrm{~m}$ decline [17-20]. The UCSD SOBQ cut-off of 20 points is well above the MCID estimate of 8 points for UCSD SOBQ in patients with IPF [21]. Treatment-emergent death was recorded as death due to any cause during the period from randomisation to 28 days after receiving the last dose of the study drug.

Time-to-event analysis of disease progression for FVC, 6MWD or UCSD SOBQ was based on a decline category with confirmation at a subsequent assessment. The outcome variables were compared between subgroups using the log-rank test with subgroup as the only term in the model, stratified (fixed effects) by study (PIPF-004, PIPF-006 and PIPF-016) and geographic region (USA and the rest of the world). A proportional hazards model was used to estimate the hazard ratio for categorical disease progression between baseline stages of lung function.

Treatment-by-subgroup interaction was tested based upon a rank analysis of covariance model, with standardised ranked change from baseline as the outcome variable and standardised ranked baseline value of \% FVC as the covariate in the model. Other factors in the model included study, geographic region, treatment group, lung function subgroup and treatment-by-subgroup interaction as fixed effects. Deaths were ranked according to time until death, where earlier incidences of death were ranked worse than later-occurring deaths.

Sensitivity analyses evaluated the linear slope of the change in FVC (both \% pred and L) without imputation through month 12 by treatment group and baseline FVC/GAP index stage subgroups.

The standardised mean difference (defined as mean difference divided by standard deviation of the difference) was assessed following the methods described in HigGins and GreEN [22].

\section{Results}

\section{Baseline characteristics}

In this post hoc analysis, data were pooled from 1247 patients who were randomised to pirfenidone 2403 mg.day ${ }^{-1}$ or placebo in the phase 3 CAPACITY and ASCEND trials. Patients were categorised using baseline FVC ( $\geqslant 80 \%$ ( $n=146$ and $n=170$ in the pirfenidone and placebo groups, respectively) and $<80 \%$ $(\mathrm{n}=477$ and $\mathrm{n}=454$, respectively)) or GAP index stage (GAP stage I ( $\mathrm{n}=247$ and $\mathrm{n}=235$, respectively) and II-III ( $n=376$ and $n=387$, respectively)). The GAP index stage could not be calculated for two patients due to missing data.

Baseline demographic and clinical characteristics were generally similar across all four groups (table 1), with no unexpected differences between patients with FVC $\geqslant 80 \% / G A P$ stage I versus FVC $<80 \% / G A P$ stage II-III.

\section{Disease progression by FVC and GAP index category}

Disease progression was assessed over 12 months in the pooled placebo population. Overall, patients with both more preserved and less preserved lung function demonstrated clinically significant disease progression at 12 months in terms of categorical declines in FVC, 6MWD and dyspnoea scores (online supplementary table S1). The proportion of patients with baseline FVC $<80 \%$ who experienced a decline of $\geqslant 10 \%$ FVC or death over 12 months was not statistically different from those with baseline FVC $\geqslant 80 \%$, (hazard ratio 1.28 (95\% CI $0.85-1.92$ ), $\mathrm{p}=0.2403$; figure 1a). In contrast, the likelihood of experiencing a decline of $\geqslant 50 \mathrm{~m}$ in $6 \mathrm{MWD}$ (or death) or a change of $\geqslant 20$ points in the UCSD SOBQ total score (or death) was significantly greater in patients with baseline FVC $<80 \%$ compared with patients with baseline FVC $\geqslant 80 \%$ (6MWD hazard ratio 1.67 (95\% CI 1.16-2.41), $\mathrm{p}=0.0049$ and UCSD SOBQ hazard ratio 2.68 (95\% CI 1.71-4.21), $\mathrm{p}<0.0001)$. 
TABLE 1 Baseline demographic and clinical characteristics

\begin{tabular}{|c|c|c|c|c|c|c|c|c|}
\hline & \multicolumn{2}{|c|}{ Baseline FVC $\geqslant 80 \%$} & \multicolumn{2}{|c|}{ Baseline FVC $<80 \%$} & \multicolumn{2}{|c|}{ Baseline GAP stage I } & \multicolumn{2}{|c|}{ Baseline GAP stage II-III } \\
\hline & Pirfenidone & Placebo & Pirfenidone & Placebo & Pirfenidone & Placebo & Pirfenidone & Placebo \\
\hline Patients & 146 & 170 & 477 & 454 & 247 & 235 & 376 & 387 \\
\hline Male & $102(69.9)$ & $122(71.8)$ & $361(75.7)$ & $343(75.6)$ & $136(55.1)$ & $135(57.4)$ & $327(87.0)$ & 328 (84.8) \\
\hline White & 136 (93.2) & $160(94.1)$ & $456(95.6)$ & $430(94.7)$ & 231 (93.5) & $222(94.5)$ & $361(96.0)$ & $366(94.6)$ \\
\hline BMI $\mathrm{kg} \cdot \mathrm{m}^{-2}$ & $29.4 \pm 4.2$ & $29.4 \pm 4.3$ & $30.0 \pm 4.5$ & $29.8 \pm 4.5$ & $30.3 \pm 4.9$ & $30.5 \pm 4.6$ & $29.6 \pm 4.1$ & $29.2 \pm 4.2$ \\
\hline Ex & $94(64.4)$ & $109(64.1)$ & $312(65.4)$ & $275(60.6)$ & $147(59.5)$ & $136(57.9)$ & $259(68.9)$ & 247 (63.8) \\
\hline Current & $5(3.4)$ & $9(5.3)$ & $3(0.6)$ & $8(1.8)$ & $7(2.8)$ & $10(4.3)$ & $1(0.3)$ & $7(1.8)$ \\
\hline Supplemental $\mathbf{O}_{2}$ use & $20(13.7)$ & 27 (15.9) & 135 (28.3) & $123(27.1)$ & $54(21.9)$ & $48(20.4)$ & $101(26.9)$ & $102(26.4)$ \\
\hline FVC \% pred & $89.5 \pm 9.2$ & $89.6 \pm 9.2$ & $66.2 \pm 8.6$ & $65.4 \pm 8.0$ & $76.9 \pm 13.9$ & $77.8 \pm 14.3$ & $68.1 \pm 11.5$ & $68.3 \pm 11.6$ \\
\hline D.co \% & $51.0 \pm 10.5$ & $50.3 \pm 11.3$ & $43.9 \pm 9.5$ & $43.8 \pm 10.5$ & $51.2 \pm 10.4$ & $51.5 \pm 10.4$ & $41.8 \pm 8.1$ & $42.0 \pm 10.0$ \\
\hline FEV $1 / F V C$ ratio & $0.82 \pm 0.05$ & $0.81 \pm 0.05$ & $0.84 \pm 0.04$ & $0.84 \pm 0.04$ & $0.84 \pm 0.05$ & $0.83 \pm 0.05$ & $0.84 \pm 0.04$ & $0.83 \pm 0.05$ \\
\hline Probable/possible UIP & $9(6.2)$ & $7(4.1)$ & $37(7.8)$ & $33(7.3)$ & $29(11.7)$ & $20(8.5)$ & $17(4.5)$ & $20(5.2)$ \\
\hline \multicolumn{9}{|l|}{ SLB diagnosis } \\
\hline Definite UIP & 55 (90.2) & 59 (90.8) & $174(84.9)$ & $161(83.4)$ & $114(87.7)$ & 102 (84.3) & $115(84.6)$ & 116 (85.9) \\
\hline Probable/possible UIP & $6(9.8)$ & $6(9.2)$ & $29(14.1)$ & 30 (15.5) & $14(10.8)$ & $18(14.9)$ & $21(15.4)$ & 18 (13.3) \\
\hline $\begin{array}{l}\text { Time from IPF diagnosis } \\
\text { to randomisation years }\end{array}$ & $1.4 \pm 1.1$ & $1.4 \pm 1.1$ & $1.5 \pm 1.1$ & $1.5 \pm 1.1$ & $1.3 \pm 1.0$ & $1.3 \pm 1.1$ & $1.5 \pm 1.1$ & $1.6 \pm 1.1$ \\
\hline
\end{tabular}

Data are presented as $\mathrm{n}$, mean \pm SD or $\mathrm{n}(\%)$. FVC: forced vital capacity; GAP: gender, age and physiology index; BMI: body mass index; DLCO: diffusing capacity of the lung for carbon monoxide; FEV1: forced expiratory volume in $1 \mathrm{~s}$; 6MWD: 6-min walking distance; UCSD SOBQ: University of California San Diego Shortness of Breath Questionnaire; HRCT: high-resolution computed tomography; UIP: usual interstitial pneumonia; SLB: surgical lung biopsy; IPF: idiopathic pulmonary fibrosis.

Similar observations were made when categorising the patients by baseline GAP index stage (figure $1 \mathrm{~b}$ ). The likelihood of experiencing a decline of $\geqslant 10 \%$ FVC or death (hazard ratio 1.34 (95\% CI 0.93-1.94), $\mathrm{p}=0.1175$ ) over 12 months was not statistically different between patients with GAP stage I and II-III disease; however, patients with GAP stage II-III disease were significantly more likely to experience a $\geqslant 50 \mathrm{~m}$ decline in $6 \mathrm{MWD}$ or death (hazard ratio 1.77 (95\% CI 1.28-2.45), $\mathrm{p}=0.0004$ ) or a $\geqslant 20$ point worsening in the UCSD SOBQ total score or death (hazard ratio 2.32 (95\% CI 1.61-3.33), p<0.0001) compared with patients with GAP stage I disease.

\section{Treatment effect of pirfenidone}

Treatment effect of pirfenidone versus placebo

As observed in the pooled placebo population, patients in the pooled pirfenidone population with less preserved (FVC $<80 \% / G A P$ stage II-III) and more preserved (FVC $\geqslant 80 \% / G A P$ stage I) baseline lung function demonstrated a clinically significant categorical worsening in FVC, 6MWD and UCSD SOBQ scores at 12 months (online supplementary table S2). The proportion of patients in each lung function subgroup experiencing categorical declines above a given threshold for different efficacy outcomes was similar across classification methods (FVC versus GAP index).

Pirfenidone treatment reduced the decline in categorical and absolute FVC, 6MWD and UCSD SOBQ scores at 12 months compared with placebo (figure 2). Patients treated with pirfenidone were significantly less likely to have $\mathrm{a} \geqslant 10 \%$ decline in FVC or death over 12 months compared with patients who received placebo ( $\mathrm{p}<0.0001$ for all subgroups). Pirfenidone-treated patients in the GAP stage I, GAP stage II-III and $\mathrm{FVC}<80 \%$ subgroups at baseline were also less likely to experience a $\geqslant 50 \mathrm{~m}$ decline in $6 \mathrm{MWD}$ or death compared with placebo-treated patients; however, the treatment effect of pirfenidone did not reach statistical significance in patients with baseline FVC $\geqslant 80 \%(p=0.0514)$.

Compared with placebo patients, pirfenidone-treated patients with FVC $<80 \% / G A P$ stage II-III were significantly less likely to experience a $\geqslant 20$ point worsening of UCSD SOBQ scores or death at 12 months (for FVC <80\%, p=0.0096; for GAP stage II-III, p=0.0053). Conversely, in patients with baseline FVC $\geqslant 80 \%$ or GAP stage I disease, the relative difference in the proportion of pirfenidone- versus 


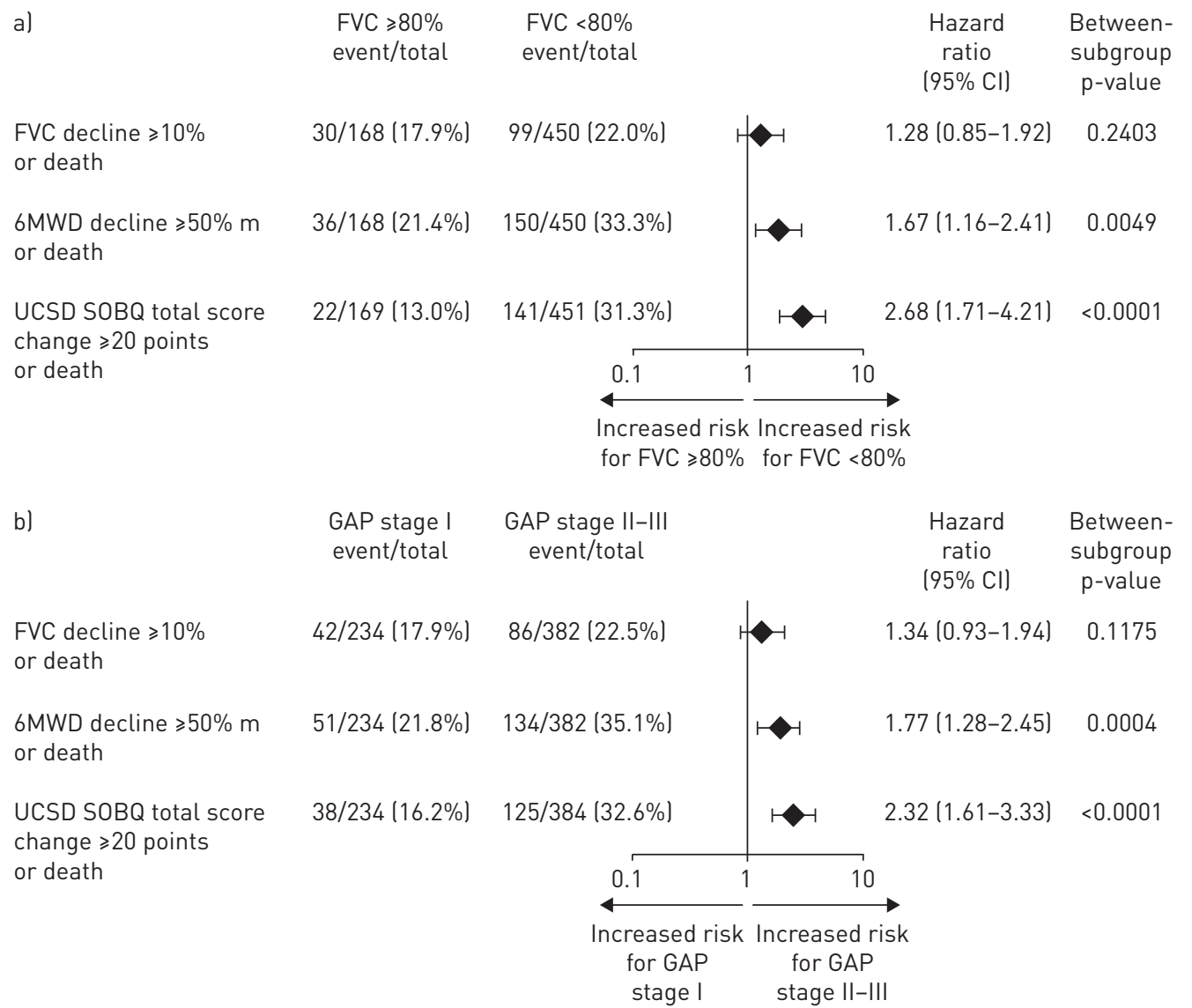

FIGURE 1 Risk of disease progression in the pooled placebo population by baseline stage of lung function according to a) forced vital capacity (FVC) and b) GAP (gender, age and physiologyl index. 6MWD: 6-min walking distance; UCSD SOBQ: University of California San Diego Shortness of Breath Questionnaire.

placebo-treated patients experiencing $a \geqslant 20$ point worsening of UCSD SOBQ scores or death at 12 months was not significant $(\mathrm{p}=0.8929$ and $\mathrm{p}=0.9767$, respectively). These results indicate that pirfenidone treatment in the subgroup of patients with more preserved lung function at baseline did not have a significant treatment benefit on dyspnoea scores.

\section{Standardised treatment effect of pirfenidone by baseline lung function}

Pirfenidone effectively reduced disease progression at 12 months as assessed by changes in different functional measures in both the baseline FVC $<80 \% / G A P$ stage II-III and FVC $\geqslant 80 \% / G A P$ stage I subgroups, with no significant differences between the groups (figure 2). The magnitude of pirfenidone treatment effect on reducing categorical FVC decline or death was similar in both FVC $<80 \% / G A P$ stage II-III and FVC $\geqslant 80 \% / G A P$ stage I subgroups (treatment-by-subgroup interaction p-values: FVC $p=0.3969$; GAP index $p=0.8152$ ). Sensitivity analyses performed without imputation confirmed the positive treatment effect of pirfenidone versus placebo on change in FVC through 12 months, with larger treatment effects generally observed in patients with FVC $\geqslant 80 \% / G A P$ stage I (online supplementary table S3).

Similarly, pirfenidone consistently reduced the categorical decline in 6MWD or death in both FVC $<80 \% /$ GAP stage II-III and FVC $\geqslant 80 \% / G A P$ stage I subgroups, with comparable magnitude of treatment effect (treatment-by-subgroup p-values: FVC p=0.9583; GAP index $\mathrm{p}=0.9327$ ). Also, the treatment effect of pirfenidone on reducing the proportion of patients experiencing a categorical worsening of dyspnoea or death was not statistically significantly different for the FVC $<80 \% / G A P$ stage II-III versus the FVC $\geqslant 80 \% / G A P$ stage I subgroups (treatment-by-subgroup interaction p-values: FVC p=0.1957; GAP index p=0.0804).

\section{Safety outcomes}

Overall, the incidence of treatment-emergent adverse events was mostly similar across baseline stages of lung function (table 2). Most events were mild to moderate in intensity, with no meaningful differences in treatment-emergent adverse events between the pirfenidone groups; however, grade 4 treatment-emergent 


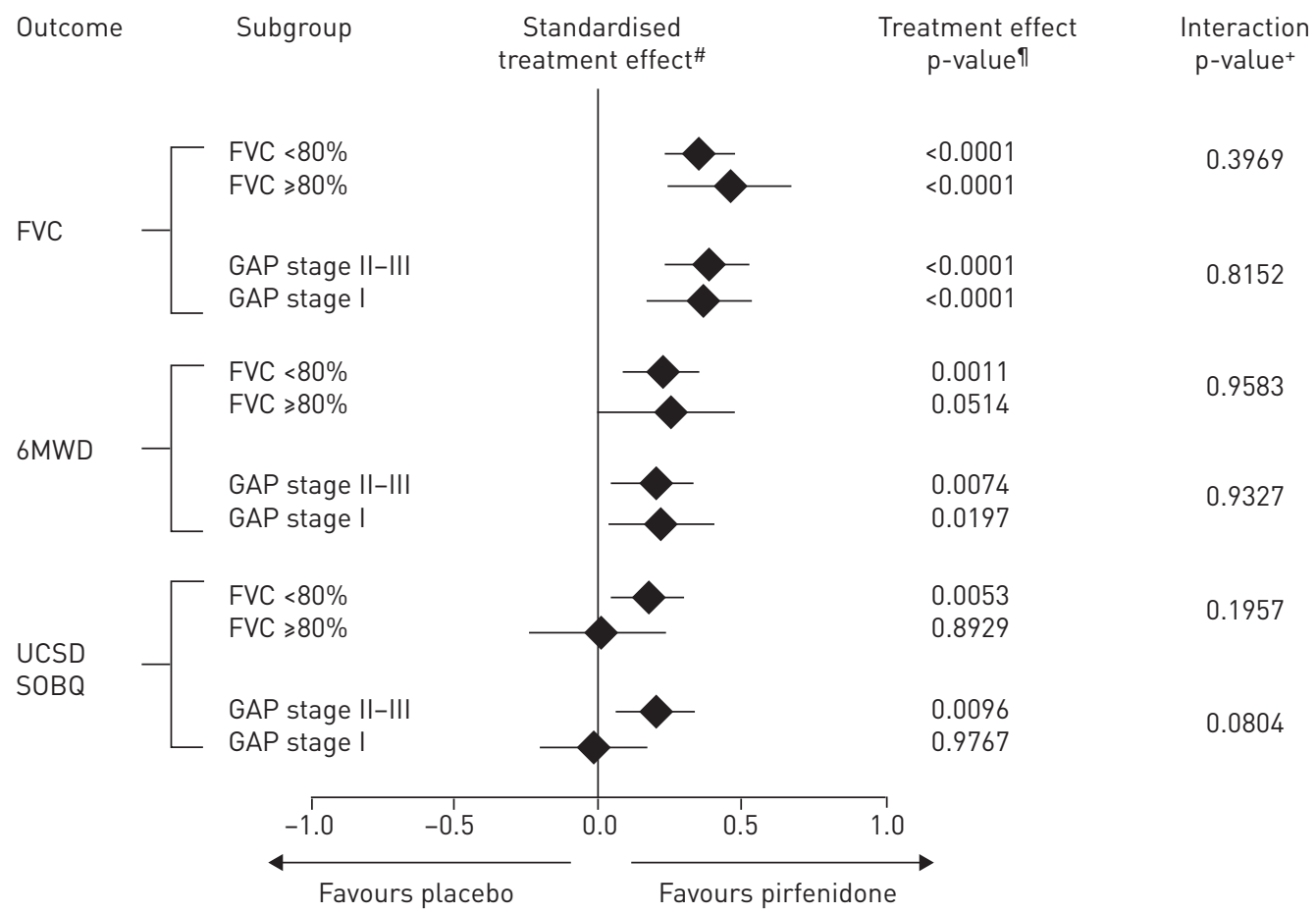

FIGURE 2 Treatment effect of pirfenidone by baseline stage of lung function. FVC: forced vital capacity; GAP: gender, age and physiology index; 6MWD: 6-min walking distance; UCSD SOBQ: University of California San Diego Shortness of Breath Questionnaire. \#: for FVC and 6MWD: treatment difference=pirfenidoneplacebo; for UCSD SOBQ, treatment difference=placebo-pirfenidone. ": $p$-values represent categorical difference (pirfenidone versus placebo); percentage relative treatment difference in the proportion of patients achieving efficacy outcomes were calculated using the formula: $100 \times\left(\right.$ pirfenidone-placebo)/placebo. ${ }^{+}$: $p$-value is from the test statistic for testing the interaction between the treatment and subgroup variable.

adverse events were more frequent in the GAP stage II-III versus I group in the placebo population (7.2\% versus $2.6 \%$ ). Treatment-emergent adverse events leading to discontinuation and treatment-emergent serious adverse events were more frequent in patients with less preserved lung function in both pirfenidone and placebo groups.

The most common treatment-emergent adverse events (observed in $\geqslant 10 \%$ of patients in either treatment group) included gastrointestinal and respiratory events and skin rashes (table 3). Overall, there were no differences in the type of adverse events reported in patients with baseline FVC $<80 \% / G A P$ stage II-III disease versus patients with baseline $\mathrm{FVC} \geqslant 80 \% / \mathrm{GAP}$ stage I disease.

\begin{tabular}{|c|c|c|c|c|c|c|c|c|}
\hline Safety event & Pirfenidone & Placebo & Pirfenidone & Placebo & Pirfenidone & Placebo & Pirfenidone & Placebo \\
\hline Patients & 146 & 170 & 477 & 454 & 247 & 235 & 376 & 387 \\
\hline $\begin{array}{l}\text { Any treatment-emergent } \\
\text { adverse event }\end{array}$ & 140 (95.9) & 163 (95.9) & $475(99.6)$ & 439 (96.7) & $240(97.2)$ & 225 (95.7) & 375 (99.7) & 375 (96.9) \\
\hline $\begin{array}{l}\text { Any treatment-emergent } \\
\text { adverse event leading to } \\
\text { discontinuation }\end{array}$ & $10(6.8)$ & $10(5.9)$ & $64(13.4)$ & 44 (9.7) & 24 (9.7) & $10(4.3)$ & 50 (13.3) & $44(11.4)$ \\
\hline $\begin{array}{l}\text { Any treatment-emergent } \\
\text { serious adverse event }\end{array}$ & $27(18.5)$ & $27(15.9)$ & $101(21.2)$ & $112(24.7)$ & 50 (20.2) & $34(14.5)$ & $78(20.7)$ & $105(27.1)$ \\
\hline Treatment-emergent death & $2(1.4)$ & $3(1.8)$ & $12(2.5)$ & $29(6.4)$ & $3(1.2)$ & $2(0.9)$ & $11(2.9)$ & $30(7.8)$ \\
\hline
\end{tabular}

Data are presented as $\mathrm{n}$ or $\mathrm{n}(\%)$. FVC: forced vital capacity; GAP: gender, age and physiology index. " : occurring through 12 months during treatment period (from the first dose up to 28 days after the last dose of study drug). 
TABLE 3 Summary of most commonly occurring treatment-emergent adverse events (incidence $\geqslant 10 \%$ in either group)

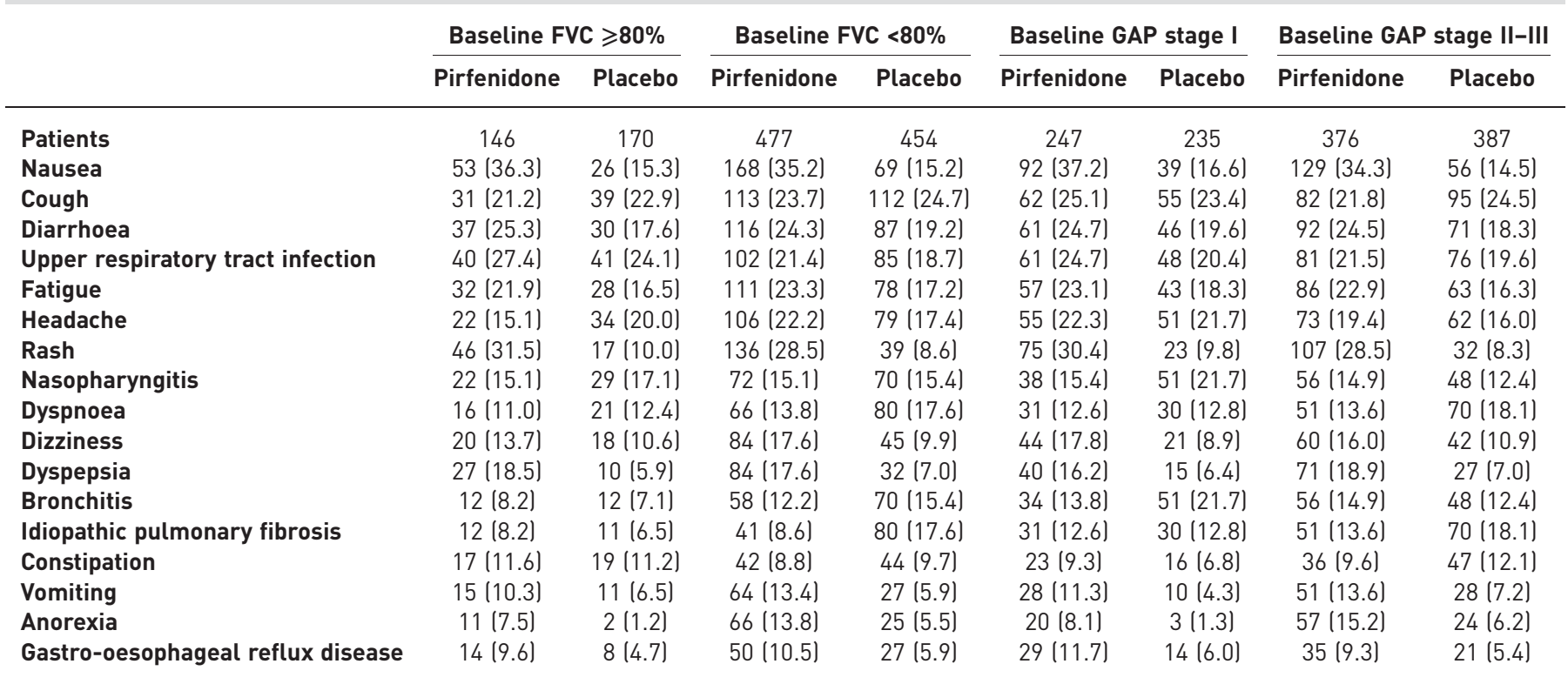

Data are presented as $\mathrm{n}$ or $\mathrm{n}(\%)$. FVC: forced vital capacity; GAP: gender, age and physiology index.

\section{Discussion}

This post hoc analysis used pooled data from patients randomised to pirfenidone $2403 \mathrm{mg}^{-\mathrm{day}^{-1}}$ or placebo in three phase 3 controlled trials to assess disease progression and the treatment effect of pirfenidone in patients categorised by baseline stage of lung function. While all patients demonstrated a decline in FVC, 6MWD and dyspnoea at 12 months, irrespective of stage of lung function at baseline, treatment outcomes were better in patients randomised to pirfenidone versus placebo. The effect of pirfenidone treatment on reduction of categorical FVC and 6MWD decline at 12 months was generally consistent and statistically significant across subgroups, regardless of whether baseline lung function was defined using FVC or GAP index stage. Only patients with FVC $<80 \%$ or GAP stage II-III at baseline experienced a significant treatment effect with pirfenidone on reducing worsening of dyspnoea at 12 months as compared with placebo. However, the treatment-by-subgroup interaction demonstrated that there were no statistically significant differences in treatment effect on dyspnoea in the FVC <80\%/GAP stage II-III subgroups versus the FVC $\geqslant 80 \% / G A P$ stage I subgroups, suggesting that dyspnoea scores may not provide useful end-point information in patients with milder lung impairment.

There is no consensus on how to classify disease severity in patients with IPF. In this analysis, FVC and GAP index were used as two separate methods of categorising patients as having more preserved versus less preserved lung function at baseline, with the understanding that baseline FVC values may not accurately represent severity of impairment relative to pre-morbid values (i.e. a baseline value of $85 \%$ may still be "severe" for a patient with a pre-morbid value of 130\%). Generally, FVC is considered to be the most reliable measure of disease progression, with less intra- and intersubject variability than the 6MWD and UCSD SOBQ $[6-8,16,23]$. A decline of $5 \%$ in FVC is prognostically important, and a decline of $\geqslant 10 \%$ suggests an increased risk of death within 12 months $[6,16,24]$. A retrospective analysis of patients with IPF compared the prognostic value of using relative versus absolute changes to identify patients with $\mathrm{a} \geqslant 10 \%$ decline in FVC and found that relative changes in FVC may be more sensitive [7]; however, evidence-based guidelines for the management of IPF suggest that measuring an absolute decline $\geqslant 10 \%$ in FVC (as performed in the current analysis) is an acceptable method of assessing disease severity and estimating the risk of mortality [2]. The same guidelines also consider an absolute decline $\geqslant 15 \%$ in DLCO as a surrogate marker of mortality and a potential indicator of disease progression [2]. However, DLCO was only measured at baseline in the PIPF-016 study and was not significantly altered by pirfenidone in the PIPF-004 and PIPF-006 studies [12, 13]; therefore, the use of DLCO would not have been feasible nor sensitive enough to detect disease progression with pirfenidone treatment in the current analysis.

A recent study examined the consistency of the effect of nintedanib across a range of IPF phenotypes through various pre-specified subgroup analyses of pooled data from the phase 3 clinical trials, including stratification by baseline FVC $\%$ pred $(\leqslant 70 \%$ versus $>70 \%)$ [25]. The authors concluded that the treatment 
effect of nintedanib was consistent across all pre-specified subgroups, which is in agreement with the recently published subgroup analysis of the treatment effect of pirfenidone on FVC decline using pooled data from the phase 3 clinical trials [26]. Both studies strengthen the data in the current analysis and provide further support that treatment for IPF is beneficial regardless of baseline demographic or clinical characteristics.

A number of limitations should be considered in light of these results. First, this was a post hoc pooled analysis of three phase 3 studies with slight differences in study design (e.g. different durations of treatment, primary efficacy end-points assessed at different times and slightly different inclusion criteria). The analysis was not powered to assess differences in the categorical efficacy outcomes by different stages of lung function. Second, the comparisons between subgroups in this analysis should be interpreted with caution because there were more patients with less preserved lung function at baseline (particularly when assessed by FVC method) than more preserved lung function. It should also be noted that patient characteristics in this analysis were defined by the inclusion criteria of the individual phase 3 studies (e.g. FVC $50-90 \%$ and DLCO $30-90 \%$ in ASCEND and FVC $50-90 \%$ and DLCO $35-90 \%$ in CAPACITY, respectively) and may not reflect patients with IPF seen in clinical practice, who may have more or less impaired lung function, or who may present with more comorbidities. Third, we did not analyse how individual components of the GAP index may influence the categorisation of patients as having GAP stage I versus II-III disease, i.e. further analyses were not performed to assess whether age, sex or physiological factors (FVC or DLCO) were the main drivers of GAP index stages. Despite the underrepresentation of patients with GAP stage III disease, likely attributed to the exclusion of patients with severe disease (FVC $<50 \%$ and/or DLCO $<30 \%$ or $<35 \%$ ) from the clinical trials, GAP staging and subsequent analysis of outcomes by GAP stage I versus II-III is still valuable, because it is a commonly used predictor for future mortality. Finally, it should be emphasised that there are no universal definitions for different subgroups of disease severity. Other analyses and publications may refer to "mild" and "moderate" disease, with different cut-off points for each subgroup; however, these values have not been validated in IPF populations. Furthermore, the terms "mild" and "moderate" may not be appropriate in the context of an irreversible, progressive disease, while "early" and "late/advanced" disease may be difficult to characterise in the context of an unpredictable disease that may not be diagnosed right away.

Overall, in this pooled phase 3 clinical trial population of patients with IPF, clinically significant disease progression occurred in patients with both more preserved and less preserved lung function at baseline, regardless of whether this was classified by FVC or GAP index stage. Likewise, the magnitude of pirfenidone treatment effect on functional measures was comparable between the FVC $\geqslant 80 \%$ versus $<80 \%$ and the GAP stage I versus II-III subgroups. Taken together, these findings support the initiation of treatment with pirfenidone, irrespective of stage of lung function at baseline; however, as recommended by the current clinical practice guidelines, shared decision making between clinician and patient is encouraged when choosing the most appropriate therapy [27].

\section{Acknowledgements}

The authors would like to thank Helen Tang (InterMune Inc., Brisbane, CA, USA) for conducting the statistical analyses for this manuscript, and Zhengning Lin and David Kardatzke (InterMune Inc., Brisbane, CA, USA) for their contribution to the statistical inputs. Support for third-party writing assistance for this manuscript, furnished by Eric Deutsch (Health Interactions, Hamilton, NJ, USA), was provided by F. Hoffmann-La Roche Ltd. Elizabeth A. Fagan and Eduard Gorina were at InterMune Inc. (Brisbane, CA, USA) at the time of writing; InterMune Inc. became a wholly owned Roche subsidiary in 2014.

\section{References}

1 Ley B, Collard HR, King TE Jr. Clinical course and prediction of survival in idiopathic pulmonary fibrosis. Am J Respir Crit Care Med 2011; 183: 431-440.

2 Raghu G, Collard HR, Egan JJ, et al. An official ATS/ERS/JRS/ALAT statement: idiopathic pulmonary fibrosis: evidence-based guidelines for diagnosis and management. Am J Respir Crit Care Med 2011; 183: 788-824.

3 du Bois RM. An earlier and more confident diagnosis of idiopathic pulmonary fibrosis. Eur Respir Rev 2012; 21: 141-146.

4 Kim DS, Collard HR, King TE Jr. Classification and natural history of the idiopathic interstitial pneumonias. Proc Am Thorac Soc 2006; 3: 285-292.

5 Kim HJ, Perlman D, Tomic R. Natural history of idiopathic pulmonary fibrosis. Respir Med 2015; 109: 661-670.

6 du Bois RM, Weycker D, Albera C, et al. Forced vital capacity in patients with idiopathic pulmonary fibrosis: test properties and minimal clinically important difference. Am J Respir Crit Care Med 2011; 184: 1382-1389.

7 Richeldi L, Ryerson CJ, Lee JS, et al. Relative versus absolute change in forced vital capacity in idiopathic pulmonary fibrosis. Thorax 2012; 67: 407-411.

8 Wells AU. Forced vital capacity as a primary end point in idiopathic pulmonary fibrosis treatment trials: making a silk purse from a sow's ear. Thorax 2013; 68: 309-310.

9 Kolb M, Collard HR. Staging of idiopathic pulmonary fibrosis: past, present and future. Eur Respir Rev 2014; 23 : 220-224. 
10 Ley B, Ryerson CJ, Vittinghoff E, et al. A multidimensional index and staging system for idiopathic pulmonary fibrosis. Ann Intern Med 2012; 156: 684-691.

11 Salisbury ML, Xia M, Zhou Y, et al. Idiopathic pulmonary fibrosis: Gender-Age-Physiology index stage for predicting future lung function decline. Chest 2016; 149: 491-498.

12 King TE Jr, Bradford WZ, Castro-Bernardini S, et al. A phase 3 trial of pirfenidone in patients with idiopathic pulmonary fibrosis. N Engl J Med 2014; 370: 2083-2092.

13 Noble PW, Albera C, Bradford WZ, et al. Pirfenidone in patients with idiopathic pulmonary fibrosis (CAPACITY): two randomised trials. Lancet 2011; 377: 1760-1769.

14 Lederer DJ, Bradford WZ, Fagan EA, et al. Sensitivity analyses of the change in FVC in a phase 3 trial of pirfenidone for idiopathic pulmonary fibrosis. Chest 2015; 148: 196-201.

15 Eakin EG, Resnikoff PM, Prewitt LM, et al. Validation of a new dyspnea measure: the UCSD Shortness of Breath Questionnaire. University of California, San Diego. Chest 1998; 113: 619-624.

16 du Bois RM, Weycker D, Albera C, et al. Ascertainment of individual risk of mortality for patients with idiopathic pulmonary fibrosis. Am J Respir Crit Care Med 2011; 184: 459-466.

17 du Bois RM, Weycker D, Albera C, et al. Six-minute-walk test in idiopathic pulmonary fibrosis: test validation and minimal clinically important difference. Am J Respir Crit Care Med 2011; 183: 1231-1237.

18 Swigris JJ, Wamboldt FS, Behr J, et al. The 6 minute walk in idiopathic pulmonary fibrosis: longitudinal changes and minimum important difference. Thorax 2010; 65: 173-177.

19 Nathan SD, du Bois RM, Albera C, et al. Validation of test performance characteristics and minimal clinically important difference of the 6-minute walk test in patients with idiopathic pulmonary fibrosis. Respir Med 2015; 109: 914-922.

20 du Bois RM, Albera C, Bradford WZ, et al. 6-Minute walk distance is an independent predictor of mortality in patients with idiopathic pulmonary fibrosis. Eur Respir J 2014; 43: 1421-1429.

21 Swigris JJ, Han M, Vij R, et al. The UCSD shortness of breath questionnaire has longitudinal construct validity in idiopathic pulmonary fibrosis. Respir Med 2012; 106: 1447-1455.

22 Higgins JPT, Green S. Cochrane Handbook for Systematic Reviews of Interventions. Version 5.1.0 [updated March 2011]. The Cochrane Collaboration, 2011. www.cochrane-handbook.org Date last accessed: November 17, 2015.

23 Nathan SD, Meyer KC. IPF clinical trial design and endpoints. Curr Opin Pulm Med 2014; 20: 463-471.

24 Zappala CJ, Latsi PI, Nicholson AG, et al. Marginal decline in forced vital capacity is associated with a poor outcome in idiopathic pulmonary fibrosis. Eur Respir J 2010; 35: 830-836.

25 Costabel U, Inoue Y, Richeldi L, et al. Efficacy of nintedanib in idiopathic pulmonary fibrosis across prespecified subgroups in INPULSIS. Am J Respir Crit Care Med 2016; 193: 178-185.

26 Noble PW, Albera C, Bradford WZ, et al. Pirfenidone for idiopathic pulmonary fibrosis: analysis of pooled data from the three multinational phase 3 trials. Eur Respir J 2016; 47: 243-253.

27 Raghu G, Rochwerg B, Zhang Y, et al. An official ATS/ERS/JRS/ALAT clinical practice guideline: treatment of idiopathic pulmonary fibrosis. An update of the 2011 clinical practice guideline. Am J Respir Crit Care Med 2015; 192: e3-e19. 